\title{
Evolvable Production Systems: Mechatronic Production Equipment with Evolutionary Control
}

\author{
Antonio Maffei ${ }^{1}$, Mauro Onori ${ }^{1}$, Pedro Neves ${ }^{2}$, and José Barata ${ }^{2}$ \\ ${ }^{1}$ Royal Institute of Technology, Dept. Of Production Engineering, \\ Stockholm, Sweden \\ Tel.: 46-8-7906637 \\ onori@iip.kth.se \\ ${ }^{2}$ Universidade Nova de Lisboa/UNINOVA, Quinta da Torre, \\ Monte da Caparica, Caparica, Portugal \\ jab@uninova.pt
}

\begin{abstract}
Current major roadmapping efforts have all clearly underlined that true industrial sustainability will require far higher levels of systems' autonomy and adaptability. In accordance with these recommendations, the Evolvable Production Systems (EPS) has aimed at developing such technological solutions and support mechanisms. Since its inception in 2002 as a next generation of production systems, the concept is being further developed and tested to emerge as a production system paradigm. Characteristically, Evolvable systems have distributed control, and are composed of intelligent modules with embedded control. A concerted effort is being exerted through European research projects in collaboration with manufacturers, technology/equipment suppliers, and universities. After introducing EPS, this paper presents current developments and applications.
\end{abstract}

Keywords: Evolvable Production Systems, Modularity, Distributed Control.

\section{Introduction}

The major problems of manufacturing companies are all relate to uncertainty. First of all, it is very difficult for companies to predict the type and range of products that will have to be developed. The second uncertainty regards the production volumes and lifespan reached by these future products.

Evolvable systems, as a next generation of production systems, was first introduced in 2002 and has, since then, been developed and tested to emerge as a production system paradigm (see EUPASS, A3 projects). The essence of evolvability resides not only in the ability of system components to adapt to the changing conditions of operation, but also to assist in the evolution of these components in time such that processes may become self-X, $\mathrm{x}$ representing a property of the system such as reconfigurable, etc.

According to the results attained by many roadmaps, such as ManuFuture [1], ManVis [2], FutMan [3] and EUPASS [4], one of the most important objectives to be met by European industry is sustainability, which is multi-faceted: including 
economical, social and ecological aspects. The conclusion to this holistic problem is that future manufacturing solutions will have to deal with very complex scenarios.

This article gives a brief overview of the achievements attained by Evolvable Assembly Systems, EAS [5]. EAS represents one of the paradigms proposed as an opportunity to solve such threats. NOTE: the relation to Holonic Manufacturing Systems ([6], [7]) and Reconfigurable Manufacturing Systems will be described later.

Evolvable systems clearly approach the idea that system components may evolve to meet upcoming demands and needs. This inevitable bridges the fields of production engineering and computer science, but it also introduces aspects of computational evolution. Hence the link to a partial study of genetics and evolutionary principles (discussed later). Evolvable systems have characteristically distributed control, are composed of intelligent modules and are open in architecture: they go beyond the concept of embedded control and begin to suggest embedded intelligence. The technical and architectural aspects of the evolvable system development are supported by a comprehensive methodological framework. Evolvability being a system concept, it is envisaged to address every aspect of an assembly system throughout its life cycle, i.e., design and development, operation and evolution. The work has been and being implemented through large European research projects. Furthermore, integration of legacy subsystems and modules have been addressed in the methodology. It has, to date, resulted in several demonstrators and offered methodologies and architectures in support. This paper presents current developments and applications.

\section{Contribution to Technological Innovation}

As defined in [8] RMS incorporates principles of modularity, integrability, flexibility, scalability, convertibility, and diagnosability. These principles impose strong requirements to the control solution. In particular, centralized approaches become completely unsuited due to their intrinsic rigidity. Decentralised solutions must be considered that take into account the fundamental requirements of plugability of components, which includes the aspects related to dynamic addition /removal of components, as well as adaptation in the sense that the system does not need to be reprogrammed whenever a new module is added/ removed. This is a fundamental aspect behind any control solution approach to solve the defined requirements. Moreover, diagnosability also demands a decentralized approach, in particular if the manufacturing system is considered as a set of manufacturing components, each with diagnosis capability. The overall diagnosis of the system is obtained considering all the diagnosis information obtained from the individual modules. Due to these requirements, a particular and relevant aspect in the system being considered is the "intelligent" nature of its components, i.e., each component is considered as having computational power that will support individual diagnosability, dynamic plugability, adaptation, etc.

Therefore, the major challenge in the control solution is how to guarantee proper coordination and execution in a system in which both its components and working conditions can be dynamically changed. This is a challenge that needs a completely new approach and this is why in the context of EPS a solution based on concepts inspired from the Complexity Theory and Artificial Life is being developed. The next 
section covers what concepts from non traditional manufacturing research domains are being used to create truly dynamic control solutions.

Nevertheless, in the context of this paper it is important to clarify what are the big differences between the approach being proposed here and Holonic Manufacturing. The genesis of holonic manufacturing was very much a biological inspired approach and it was very close to the concepts of bionic and fractal. However, succeeding implementations along the years have drifted more and more from the original inspiration, and, in many aspects, the systems became more hierarchical.

Hence, the control approach to be developed in the context of EPS wants to go back to the basics, that is to say relying stringy on the original idea of considering each component as a distributed intelligent unit that may aggregate in order to create a complex system. In this context, concepts such as emergence and self-organisation become more and more important to be applied to new generation control solutions. Interestingly enough other researchers are proposing a self-organisation view in manufacturing enterprises [9]. However, true implementations of these new concepts within shop floor are still very few.

Considering what was stated above, one may view Evolvable Production Systems (EPS) as a development of the Holonic Manufacturing Systems (HMS) approach; however, a closer looks reveals that, although there are similarities in the exploitation and implementation phases, the paradigms differ quite substantially in their perspective (or trigger issue), and that only EPS achieves fine granularity. By granularity it is considered the level of complexity of the component that compose a manufacturing system. For instance, when a line is composed of several cells and these cells are modules that can be plugged in and out, this is thick granularity. If, on the other hand, the components that can be plugged in or out are grippers, sensors, or pneumatic cylinders, this is fine granularity. This issue is in fact a very important one in terms of distinguishing the paradigms.

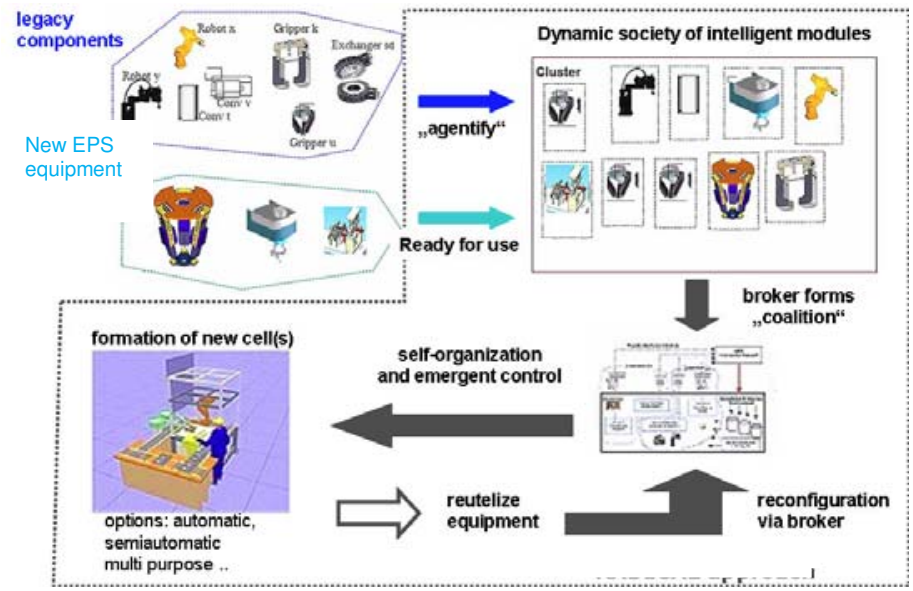

Fig. 1. Basic Overview of EPS Approach 
The main difference in the EPS paradigm is that it was created from a more dynamic, industrially-relevant perspective (trigger issue): EPS is mainly concerned with what occurs in a production system when a production change-over is called for; that is, whenever the current production system needs to undergo some change in its physical, control, or productivity layout [10]. Such changes occur at ramp-up, product change-over, or demand surges. This is where the biological inspiration to EPS first makes itself apparent: it is change that drives the adaptability/evolution of the EPS systems, not the current or known scenarios. Furthermore, as will be detailed later, the adaptability is dictated by real evolvability principles such as "survival-of-the-fittest" at algorithm level. This biological approach becomes even more evident when one studies the way modularity is achieved within EPS. In most approaches, modularity is set by either known mechanical subdivisions, or by taking the classical subdivisions that exist within manufacturing; for example, in reconfigurable assembly, the modules are most often set by the transport/handling/joining/placing/packaging processes. There is no biological link and the RMS and HMS paradigms tend to try to achieve a general, top-level solution.

EPS is radically different in this respect as it will focus on the predicted and unpredictable changes that may occur within a very limited product range (genus). The first solution will be limited and specific, and may, if successful, gradually be applied to the associated product family (species). Hence EPS is not a generic solution but a specific approach that may be adopted by other "species" if its evolutionary capabilities denote a high rate of success.

Furthermore, EPS takes a hybrid and not top-down approach to the definition of its modules. The EPS modules are defined by precise sub-processes that have been identified for a given product range: the taxonomy of the sub-processes is very detailed and therefore results in fine granularity. This is a low-level approach, and gives modules with very optimised performance characteristics: process-oriented modules [11]. Note that since it is specific, and focuses on the given evolutionary demands of a product range and its sub-processes, it may also be closely linked to product design issues. This is unique among current paradigms, which clearly underlines the contribution.

\section{Enabling Research Domains and Concepts}

The main issue to be addressed in this section is describing the areas in which EPS control systems are getting inspiration to solve the requirements for adaptability at fine granularity. Numerous scientific domains investigating phenomena which EPS also exhibit have emerged in the last few years, which can provide helpful tools and valuable theoretical background to cope with the complexity of manufacturing systems. A more detailed definition may be found in [12].

\section{Complexity Theory}

Complexity Theory looks for simple causes leading to complex behaviours [13]. Complex systems are spatially and/or temporally extended non-linear systems with many strongly-coupled degrees of freedom. They are composed of numerous in themselves often simple elements and are characterized by collective properties. EPS consist of numerous equipment modules which are connected to each other and have multi-lateral interactions. Each of them has some degrees of freedom, which are 
constraint by other system parts. Together, the modules form a system with the desired global behaviour.

\section{Artificial Life}

Taking natural life and its characteristics as an example, scientists attempt to create life-like behaviors with the capability of evolution on computers and other "artificial" media. EPS are very similar to artificial living systems, with a modifiable structure, will exhibit some kind of self-organization, can adapt to their environment, and react to stimuli. They are capable of evolving according to the circumstances, namely in terms of equipment states, and can incorporate newly available technology.

\section{Autonomic Computing}

Although at another level than the other areas described above, Autonomic Computing is a fundamental concept for EPS. The vision of Autonomic Computing [14] refers to the tendency of computers to become ubiquitous. Forming large networks and having complex and multiple interactions, they become increasingly difficult to manage. As a consequence, software will be designed to take care of itself. User interaction will be minimized and reprogramming avoided. Note that the more modules of fine granularity include computational power, the more is necessary to find new ways of coordination and automatic plugability, which is exactly what EPS want to address.

\section{Agents}

Depending on the context, an agent can be a human person, an association, an animal, or a piece of software, eventually connected to some hardware. The fundamental characteristics are identity, intelligence and the ability to act and react in order to persecute goals. Agents have at least a certain degree of autonomy and can compete or collaborate with others.

There are numerous successful experiences with agent-based systems in industry [15]. Rockwell Automation even develops agent-based systems where the agents run inside the PLC itself [16] instead of on separate computers.

\section{Self-Organization}

Reasons for implementing self-organization in EPS are to minimize and facilitate user interaction, i.e. to hide complexity and increase system autonomy. Building and configuring a system composed of numerous entities with multi-lateral interactions is a highly complex task; the more autonomy the system has, the easier it gets for the user. Agents need the capacity of organizing their collaboration themselves, in according to the needs, without passing through a central coordination point.

\section{Emergence}

Complex systems most often consist of at least two different levels: the macro-level, considering the system as a whole, and the micro-level, considering the system from the point of view of the local components. Local components behave according to local rules and based on preferably local knowledge; a representation of the entire system or knowledge about the global system functionality is neither provided by a central authority nor reachable for the components themselves. They communicate, interact with each other and exchange information. From the interaction in this local world emerge global phenomena, which are more than a straight-forward composition of the local components' behaviors and capabilities. Typically, there is not only a 
global behavior dependent on the local parts, but their behavior is also influenced by the system as a whole. Emergent phenomena are scalable, robust, and fault-tolerant, i.e. insensitive to small perturbations and local errors as well as component failure, thanks to redundancy. They exhibit graceful degradation, meaning that there is no total break-down because of minor local errors.

\section{Evolution}

EPS implies, at least partially, evolutionary behavior according to Darwinian theory. It intends to do so by applying complex adaptation behaviour at control level, and by subdiving the system equipment into specific modules. According to scientific results in the fields of Computational Ecology and Genetics ([17], [18]), the idea to adopt a modular approach is very similar to natural selection. Hence the analogy of applying modularity at system level, based on process constraints, to that of a genotypemodularity used in nature to limit the combinatorial explosion. The challenge in EPS, as with computational adaptation, is to "code" the events correctly and create the correct representations. Hence the need to study emergence and complexity theory.

\section{Architectural Aspects behind Methodology}

The EPS Methodology [19] provides the references architecture, enablers, and modeling formalisms. In the following section brief description of the methodology is given. Note that a full description of the Methodology and associated Reference Architecture is available through the EUPASS project framework (http://www.easenv.org). The EPS formalised concepts (ontology) and definitions are represented using a set of descriptive tools such as:

- Definitions of the most important concepts: module, process, product, EPS module, skills, EPS system (which is a composition of modules), etc.

- Diagrams (UML, etc) where the interactions between the concepts defined are shown. This enables to show how the EPS architecture generates assembly systems. The interaction may show the global system behaviour

The domain ontology indeed captures the concepts in the system with their specifications (consensual semantic) i.e., what the concepts are and how they are related to each other in the domain. However, it does not capture the logic behind the relationships and the how's in the synthesis and functionality of the system.

A full description of the EAS ontology, and its associated Knowledge Model, is given in [20].

\subsection{Reference Architecture, RA}

The EPS Reference Architecture (EPS_RA) describes the essential features of an Evolvable System which means the reference architecture specifies the necessary features that a system should have to be an evolvable system. The reference architecture is composed of three main elements: Principles, Technical Positions and Templates. Principles: EPS has two fundamental principles which lay the foundation and guide the development process of an evolvable system. These principles can be considered as a description of the core ideas of the evolvable system paradigm. 
Principle 1: the most innovative product design can only be achieved if no assembly process constraints are posed. The ensuing, fully independent, process selection procedure may then result in an optimal assembly system methodology.

Principle 2: Systems under dynamic conditions need to be evolvable, i.e., they need to have an inherent capability of evolution to address the new/changing requirements.

The enabling models include the development process model, the business model and the knowledge model. These models are constructed using the formalisms described above and most notably the EPS ontology. The figure below depicts the first proposed EPS_RA using IDEF0. The is a simplified high level activity model showing the main activities in the development process and their input, output, control and mechanisms needed to generate or modify instances of the architecture.

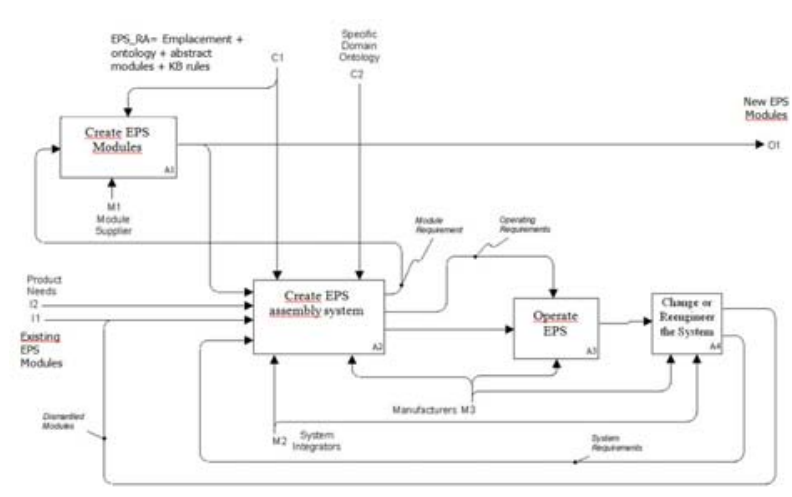

Fig. 2. The simplified EPS_RA

The traditional topdown system design is feasible only in cases where the emergent behaviour is fully describable; [21]. If emergent behaviour has to be investigated even at design and development stages, then a heterarchical or a network approach are the options.

Concepts that are formalised in the EPS Ontology are used to capture the stakeholders understanding of their own domain. The EPS knowledge model is thus a structured and formalised collection of such knowledge capturing representations of the domains. The main objective of the EPS knowledge model is to provide an environment that supports the development and operation of evolvable systems.

The domain knowledge captured using the EPS ontology and the EPS knowledge templates are the two entities used to develop the knowledge model. The knowledge models are used among other things how each module in a system should address for a new set of conditions.

The elements in the EPS knowledge model consists of the knowledge domains:

1. The enterprise knowledge domain - globalizes knowledge of the system and represents the business, organizational and global knowledge models. Enterprise knowledge enables environment recognition and maintenance of associations.

2. The product knowledge domain - captures the knowledge related to product specification and design to assembly tasks.

3. The execution knowledge domain: capturing knowledge elements related to communications, planning and scheduling

4. The learning knowledge domain: containing knowledge elements that are used to incorporate case based reasoning. 
The EPS RA is ultimately be viewed from different perspectives addressing the different concerns of the stakeholders. The stakeholders include:

- those who build the system (structure and communication views),

- those who use it (functional view),

- those who are concerned with control (control view).

\section{Practical Developments}

Initial evaluations were carried out in the test cell shown below (fig.3.0a). More industrially viable evaluations are currently being deployed within a new system being developed at KTH (fig.3.0b). For obvious reasons, reconfigurability may be illustrated by videos and is more arduous via text-details given in [22] and detailed (videos, etc.) on www.Eas-Env.Org.

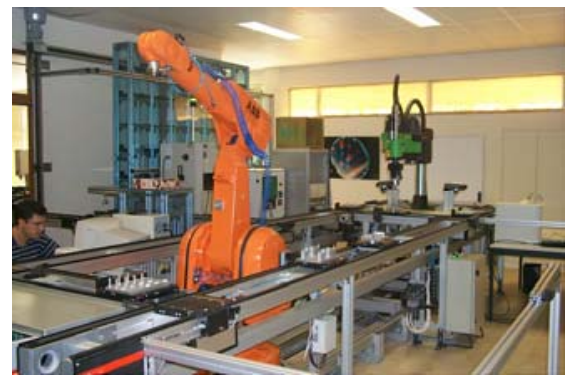

(a)

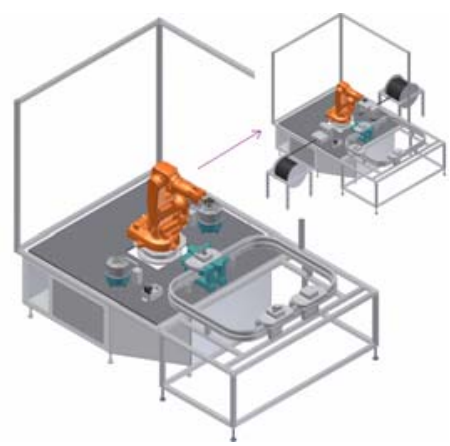

(b)

Fig. 3. (a) The Evaluation Test Case. (b) Industrial Test Case.

At present the EPS paradigm is only just starting to take a practical form, and the control solution, ontologies, and methodologies only partially describe the most recent developments. These ideas are now being put into real industrial scenarios within the EUPASS project [19], and through the participation of Electrolux Home Products Italy SpA and UNINOVA. The layout given in figure $3.0 \mathrm{~b}$ is being setup for two industrial products (self-configuring $\&$ reconfiguring).

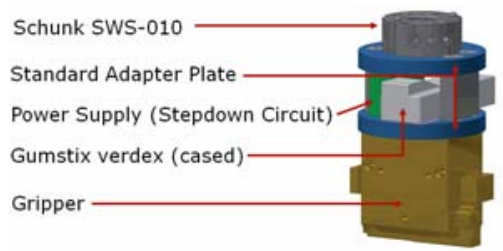

Fig. 4. The Intermodular Receptacle

The project has now demonstrated that legacy equipment may be modified to Evolvable Production System [22]. An intelligent interface was developed out of an old Schunck gripper, as shown below, and the EUPASS project has now developed a full Evolvable Production System in Windisch, Switzerland. 


\section{Conclusions}

EPS, as with other similar approaches, offers great opportunities for attaining true agility and cost-effective, stepwise automation. The technologies for achieving this are available and there are several partners willing to partake in this endeavour (for detailed list see website cited below: FESTO, BOSCH, ELECTROLUX, TQC, KIT, etc.); however, it is vital to point out that EPS does imply that the manner in which we develop and create projects for the development of assembly systems are radically changed, assuming a more synthesis-based approach.

In order to stimulate the further development and update of the paradigm and its applications, a collaborative web space has been developed, in which the architecture, standards, equipment modules and other details are detailed and made accessible: www.Eas-Env.Org. This EAS Environment web space is currently being expanded to include work from related projects.

\section{References}

1. Strategic Research Agenda-assuring the future of manufacturing in Europe; Manufuture Platform-Executive Summary, December 2005, EC (2005)

2. MANVIS, Manufacturing Visions-Integrating Diverse Perspectives into Pan-European Foresight; FP6 Support Action, NMP2-CT-2003-507139 (2006)

3. The Future of Manufacturing in Europe 2015-2020-The Challenge for Sustainability; FutMan, Institute for Prospective Technological Studies, European Commission Joint Research Centre, EUR 20705 EN (2007)

4. EUPASS Adaptive Assembly Roadmap 2015-deliverable 1.5f; Del. 1.5f, EUPASSEvolvable Ultra Precision Assembly, NMP-2-CT-2004-507978 (2008)

5. Barata, J., Onori, M., Frei, R.: ISIE 2006 -IEEE International Symposium on Industrial Electronics. IEEE, Montreal (2006)

6. Van Brussel, H., Wyns, J., Valckenaers, P., Bongaerts, L., Peeters, P.: Reference Architecture for Holonic Manufacturing Systems: PROSA. Computers in Industry 37, 255-274 (1998)

7. Bussmann, S., Mcfarlane, D.C.: Rationales for Holonic Manufacturing. In: Second Int. Workshop on Intelligent Manufacturing Systems, Leuven, Belgium (1999)

8. Elmaraghy, H.A.: Flexible and Reconfigurable Manufacturing Systems Paradigms. Int. Journal of Flexible Manufacturing Systems 17, 261-276 (2006)

9. Tharumarajah, A.: A Self-organising View of Manufacturing Enterprises. Computers in Industry 51, 185-196 (2003)

10. Maffei, A., Dencker, K.: From Flexibility to Evolvability: ways to achieve selfreconfigurability and full-autonomy. In: Proceedings of the IFAC/SYROCO 2009 Conference, Gifu, Japan (2009)

11. Maraldo, T., Onori, M., Barata, J., Semere, D.: Evolvable Assembly Systems: Clarifications and Developments to Date. In: CIRP/IWES 6th International Workshop on Emergent Synthesis, Kashiwa, Japan (2006)

12. Barata, J., Onori, M., Frei, R., Leitão, P.: Evolvable Production Systems - Enabling Research Domains. In: Proceedings of the 2nd Int. Conf. on Changeable, Agile, Reconfigurable and Virtual, Proc. CARV 2007, Toronto, Canada (2007) 
13. Delic, K.A., Dum, R.: On the Emerging Future of Complexity Sciences. ACM Ubiquity 7 (2006)

14. Kephart, J.O., Chess, D.M.: The Vision of Autonomic Computing. IEEE Computer 00189162/03, 41-50 (2003)

15. Monostori, L., Váncza, J., Kumara, S.R.T.: Agent-Based Systems for Manufacturing. CIRP Annals 55 (2006)

16. Mař́k, V., Vrba, P., Hall, K.H., Maturana, F.P.: Rockwell automation agents for manufacturing. In: AAMAS, Utrecht, NL. ACM Press, NY (2005)

17. Wagner, P.G., Altenberg, L.: Complex Adaptations and the Evolution of Evolvability. Evolution 50, 967-976

18. Bowers, C.P.: Simulating Evolution with a Computational Model of Embryogeny, PhD Thesis, The University of Birmingham (2006)

19. Lohse, N., Ferreira, P., Ratchev, S.: Multi-Agent Architecture for Self-Configuring Modular Assembly Systems. In: Proceedings of the IFAC/SYROCO 2009 Conference, Gifu, Japan (2009)

20. Ueda, K.: Journal of Artificial Intelligence in Engineering 15, 319-320 (2001)

21. Hofmann, A., Siltala, N.: Emplacement and Blue Print - An Approach to Handle and Describe Modules for Evolvable Assembly Systems. In: Proceedings of the IFAC/SYROCO 2009 Conference, Gifu, Japan (2009)

22. Adamietz, R.: Development of an Intermodular Receptacle- A First Step in Creating EAS Modules, MSC Thesis, KTH, Stockholm, Sweden (2007) 